\title{
Calibrating laser beam deflection systems for use in atomic force microscopes and cantilever sensors
}

\author{
L. Y. Beaulieu ${ }^{\text {a) }}$ \\ Department of Physics and Physical Oceanography, Memorial University, St. John's, Newfoundland, \\ Canada, AlB 37X \\ Michel Godin \\ Division of Biological Engineering, Media Laboratory, Massachusetts Institute of Technology, \\ 20 Ames Street, Cambridge, Massachusetts 02139 \\ Olivier Laroche, Vincent Tabard-Cossa, and Peter Grütter \\ Physics Department, McGill University, Montreal, Quebec, Canada, H3A 2 T8
}

(Received 10 October 2005; accepted 24 January 2005; published online 22 February 2006)

\begin{abstract}
Most atomic force microscopes and cantilever-based sensors use an optical laser beam detection system to monitor cantilever deflections. We have developed a working model that accurately describes the way in which a position sensitive photodetector interprets the deflection of a cantilever in these instruments. This model exactly predicts the numerical relationship between the measured photodetector signal and the actual cantilever deflection. In addition, the model is used to optimize the geometry of such laser deflection systems, which greatly simplifies the use of any cantilever-based instrument that uses a laser beam detection system. (C) 2006 American Institute of Physics. [DOI: 10.1063/1.2177542]
\end{abstract}

Atomic force microscopes (AFMs) and cantilever-based sensors both rely on converting a cantilever deflection into a physical quantity. For example, force sensing performed with AFMs uses the cantilever deflection to infer the amount of force applied to a sample. Cantilever-based sensors use the cantilever deflection to obtain a measure of surface stress, which then provides information on the chemical or physical phenomenon of interest.

Since the invention of the AFM, many methods have been proposed to monitor the cantilever deflection. Due to its accuracy and ease of use, the laser beam deflection system continues to be the most widely used. ${ }^{1}$ This same detection system has been used to monitor the cantilever deflection in cantilever-based sensors. ${ }^{2}$ However, due to the exquisite sensitivity of cantilever sensors, there has been a renewed interest in the laser beam deflection system. For example, there have been several papers that addressed the noise and resolution limits of the beam deflection system, ${ }^{3-5}$ while others have sought to develop a means to obtain a relationship between the cantilever deflection and the signal measured from a position-sensitive detector (PSD). ${ }^{6-11}$ Among these, it is generally accepted that for small deflections, the detector signal is directly proportional to the cantilever deflection. Some authors have proposed that the constant of proportionality is a function only of the cantilever length (CL) and the cantilever/PSD separation $(L) .^{6}$ Although the assumption of linearity is justified for small deflections, the constant of proportionality is not simply related to $\mathrm{CL}$ and/or $L$. In fact, the constant of linearity is strongly influenced by the incident laser orientation (angle of incidence $\theta$ and azimuthal angle $\phi$, see Fig. 1) and that of the photodetector. In this letter we describe a model that, as we will show, characterizes the way a laser detection system interprets the deflection of a cantilever. We will also show how this model can be used to

\footnotetext{
a) Author to whom correspondence should be addressed; electronic mail:
} beaulieu@physics.mun.ca optimize the geometry of a cantilever/laser detection system in order to establish a linear relationship between the cantilever deflection and photodetector signal. This will greatly simplify the calibration of cantilever-based instruments.

Figure 1 shows a schematic representation that describes the geometry of a cantilever/laser detection system. In this diagram, the cantilever surface is in the $x-y$ plane and is orientated in the positive $x$ direction. The incident laser hits the cantilever at a distance $D$ from the base of the cantilever chip. The incident laser is fixed at an angle of inclination $\theta$ with respect to the $x-y$ plane and at an azimuthal angle $\phi$ measured from the positive $x$ axis. The laser reflects off the free end of the cantilever and into a position-sensitive detector held at an initial distance $L$ from the cantilever. The PSD is itself inclined at an angle $\xi$ also with respect to the $x-y$ plane. In Fig. 1, the line labeled $N_{c}$ is the surface normal to the cantilever and is used to calculate the reflected laser beam direction in accordance with the law of reflection.

All calculations shown here were performed with a computer program using elementary geometric optics and standard vector geometry. ${ }^{12}$ Unlike others who have attempted to

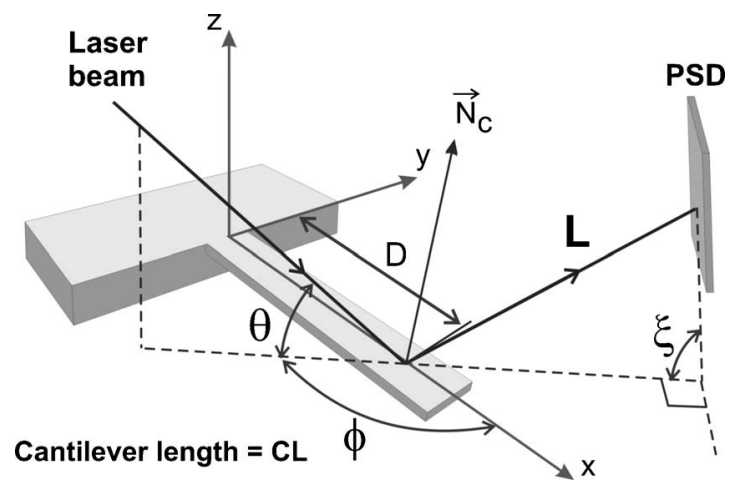

FIG. 1. Schematic representation of a laser reflecting from a cantilever into a PSD detector. 


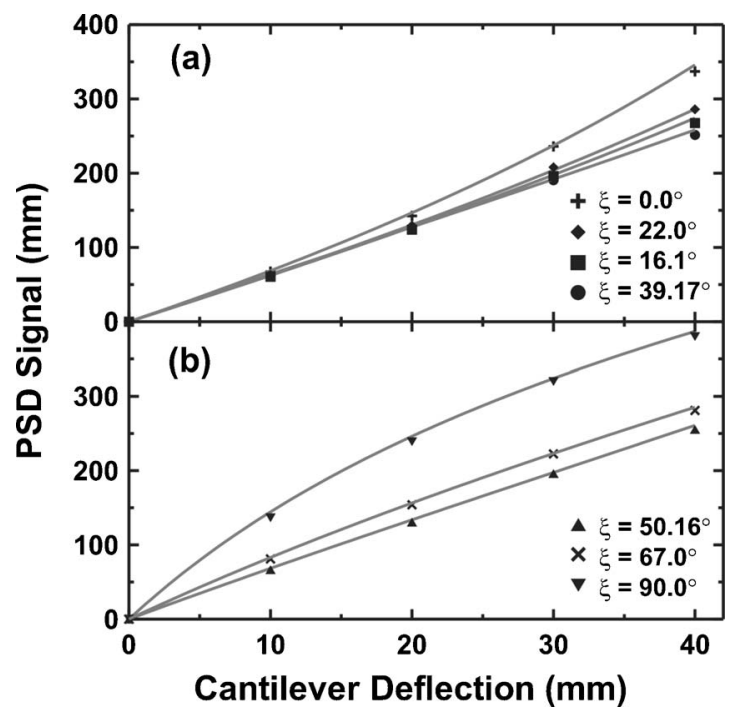

FIG. 2. (a) and (b) Comparison between experimental (symbols) and calculated data (solid lines) for the PSD signal versus cantilever deflection for different PSD detector angles $\xi$. The fixed geometrical parameters of the system were: $D=382.5 \pm 0.5 \mathrm{~mm}, L=603.7 \pm 0.5 \mathrm{~mm}, C L=410.0 \pm 0.5 \mathrm{~mm}$, $\phi=180.0 \pm 0.5^{\circ}$, and $\theta=69.9 \pm 0.5^{\circ}$.

calibrate the PSD signal/cantilever deflection, we have made no approximations of any kind..$^{6-10}$ The curvature of the cantilever was described by an end-moment deflection. ${ }^{13,14}$ As the cantilever deflects, the position of the laser beam travels on the surface of the lever. The intersection point between the incident laser and the cantilever surface was obtained numerically. The end deflection of the cantilever was also obtained numerically by solving the length equation. This procedure takes into consideration the fact that during deflections, the end point of the cantilever along the $x$ axis $\left(x_{\max }\right)$ is smaller then the total length CL of the cantilever $\left(x_{\max } \leqslant \mathrm{CL}\right)$. Taking the proper cantilever curvature into consideration is crucial for obtaining agreement with experimental results. The curvature of the cantilever not only establishes the laser/cantilever intersection point, but most importantly, dictates the reflection angle of the laser away from the surface normal (see Fig. 1). Software related to this model has been made available for download at www.physics.mun.ca/beaulieu_lab/papers/cantilever_ analysis.htm. Full details of this model are reported elsewhere. $^{15}$

From the model presented in Fig. 1, it is possible to identify different geometries, characterized by the value of $\phi$, which could be used in cantilever-based systems. While most cantilever-based sensors and AFMs are constructed with $\phi=180^{\circ}$, other geometries have been used. ${ }^{6,16}$ In this letter we focus our attention on the $\phi=180^{\circ}$ system. In order to demonstrate the accuracy of the model, a macrosized cantilever was constructed. Experiments were conducted on such a system because of the inherent ease to measure and adjust all of the parameters $(D, L, C L, \phi, \theta$, and $\xi)$ shown in Fig. 1.

Figures 2(a) and 2(b) show experimental (symbols) and calculated data (solid lines) of the measured PSD signal versus cantilever deflection for various PSD angles $\xi$. As the data show, excellent agreement is obtained between the experimental and calculated data. The PSD signal is given in units of length which describes the motion of the reflected laser spot along the PSD. We stress the fact that no adjust-

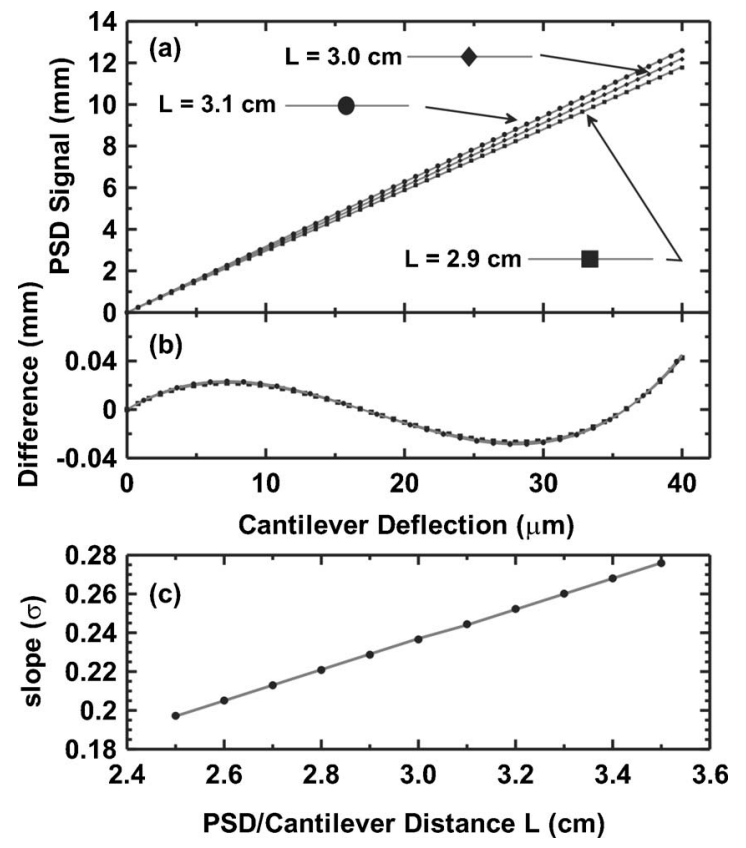

FIG. 3. (a) PSD signal versus cantilever deflection for $D=325 \mu \mathrm{m}$, CL $=375 \mu \mathrm{m}, L=2.9 \mathrm{~cm}(-\mathbf{\square}-), L=3.0 \mathrm{~cm}(-\downarrow-), L=3.1 \mathrm{~cm}(-\bigcirc-)$, $\phi=180^{\circ}, \theta=70^{\circ}$, and $\xi=36.05^{\circ}$. In this case the solid lines represent calculated data while the symbols represent linear fits. (b) Difference between the calculated data and the straight line fit for $L=2.9 \mathrm{~cm}, L=3.0 \mathrm{~cm}$, and $L$ $=3.1 \mathrm{~cm}$. (c) Slope of the optimized PSD/cantilever deflection curves versus PSD/cantilever separation $L$. Calculated data is shown by solid dots while the solid line shows a linear fit to the data.

able or fitted parameters were used to produce the calculated data. The values of $D, L, \mathrm{CL}, \phi, \theta$, and $\xi$ uniquely characterize the system. Similar measurements performed with $\phi$ $=0^{\circ}$ and $\phi=90^{\circ}$ and with different types of cantilever loading gave identical results (in accuracy). ${ }^{15}$ This model has also shown similar agreement when used to describe the motion of the laser spot on the cantilever surface upon deflection. $^{15}$

Figures 2(a) and 2(b) show how the concavity of the data changes as the value of $\xi$ is changed (similar results occur if $D, L, \phi$, and $\xi$ are fixed and $\theta$ is varied). In fact, for $\xi$ $=39.17^{\circ}$, the relationship between the PSD signal and cantilever deflection is linear. We have found that for any fixed geometry, $D, L, \phi, \theta$ (or $\xi$ ) (notably $\phi=0^{\circ}, 90^{\circ}$, or $180^{\circ}$ ), it is possible to obtain (by the method of least squares) a PSD angle $\xi$ (or incident laser angle $\theta$ ) that produces a linear relationship between the measured PSD signal and the cantilever deflection. To illustrate this, Fig. 3(a) shows calculations for a setup with the following geometry: $D=350 \mu \mathrm{m}$, $L=3.0 \mathrm{~cm}, C L=375 \mu \mathrm{m}, \phi=180^{\circ}$, and $\theta=70^{\circ}$. For this set of parameters, the optimized PSD angle, obtained by a leastsquares fit, is $\xi=36.05^{\circ}$. This geometry is characteristic of a typical cantilever sensor setup used in our laboratory. Figure 3(a) shows a comparison between calculated data (black line) and a linear fit $(\diamond)$. Figure 3(b) shows, on a much-reduced scale, the difference between the optimized linear PSD signal/cantilever deflection curve and the straight line fit.

The optimization of the beam deflection system is dependent on the cantilever deflection. The data shown in Fig. 3 was calculated for a maximum cantilever deflection of $40 \mu \mathrm{m}$. For such a large deflection, the linear fit has an average deviation of $17 \mu \mathrm{m}$ (recall the PSD signal is measured in units of length). For a more realistic maximum cantilever license or copyright; see http://apl.aip.org/about/rights_and_permissions 
deflection of $1 \mu \mathrm{m}$, the linear fit of the optimized geometry has an average deviation of only $0.0006 \mu \mathrm{m}$, which corresponds to a cantilever deflection of $0.2 \mathrm{~nm}$. Such a deflection is well within the experimental error of any cantilever based system. For a point-load type bending with a maximum cantilever deflection of $1 \mu \mathrm{m}$, the optimized geometry gives a similar average deviation of $0.0005 \mu \mathrm{m}$.

The model also shows the linearity of the PSD signal/ cantilever deflection is independent of the cantilever/PSD separation $L$, which is difficult to physically measure within 1-2 mm. Figure 3(a) shows two additional curves that illustrate how the PSD/cantilever deflection system remains linear even though the system was optimized for one specific set of variables. The first line shows calculated data (solid line) and linear fit ( $)$ for the same optimized parameters as described above with the exception that the cantilever/PSD distance has been increased to $L=3.1 \mathrm{~cm}$. In the same way, the third line shows calculated data (solid line) and linear fit (ם) for $L=2.9 \mathrm{~cm}$. The difference between the calculated data and the linear fits are shown in Fig. 3(b) (with their representative symbols), which illustrates that the difference between the optimized data $(L=3.0 \mathrm{~cm})$ and the nonoptimized data $(L=2.9$ and $3.1 \mathrm{~cm})$ are of the same magnitude.

We now show how this can be used to set up a cantilever-based instrument so as to obtain the PSD signal/ cantilever deflection relation (i.e., the slope of the straight line) without having to physically measure the PSD/ cantilever distance $L$ with an accuracy of more than $1-2 \mathrm{~mm}$.

Consider a cantilever-based instrument built to the following specifications: $D=325 \mu \mathrm{m}, \mathrm{CL}=375 \mu \mathrm{m}, \phi=180^{\circ}$, $\theta=70^{\circ}, \xi=36.05^{\circ}$, and $L=(3.0 \pm 0.2) \mathrm{cm}$. As shown in Fig. $3(\mathrm{a})$, an accuracy of $0.2 \mathrm{~cm}$ on the distance $L$ is unsatisfactory to obtain precise cantilever deflection measurements since the slope of the PSD/cantilever deflection relation varies by $7 \%$. Figure 3(c) shows how the slope of the PSD/ cantilever deflection curve changes as a function of $\mathrm{L}$ (for the optimized geometry described earlier). These data indicate that the relationship between the slope of the PSD signal/ cantilever deflection versus $L$ is also linear. To obtain the value $L$, all that is necessary is to force a cantilever to deflect by a value $\Delta X$ while monitoring the signal with the PSD. This could be, for example, the $L=3.1 \mathrm{~cm}$ line (- - ) shown in Fig. 3(a). At this point we do not know the value of $\Delta X$, which means that the slope of the curve is given by $\sigma$ $=\Delta y / \Delta X$ (where $\Delta y$ is known). Moving the detector by $\Delta L$ (in a direction parallel to the reflected laser beam) and repeating the above gives a second PSD displacement curve such as the line (- -). With these data, the value $\Delta X$ can be obtained by solving Eq. (1).

$$
\text { Slope } \sigma[\text { from Fig. 3(c) }]=\frac{\sigma_{1}-\sigma_{2}}{\Delta L}=\frac{\Delta y_{1}-\Delta y_{2}}{\Delta L \Delta X} \text {. }
$$

Once $\Delta X$ is obtained, the exact value of $L$ is then calculated from our model. In performing this calibration [Eq. (1)] two things are important. Firstly, the cantilever deflection $\Delta X$ must be the same in both cases. This can be accomplished using a piezolever or by heating a cantilever that has been coated on one side with a material that has a different coefficient of thermal expansion then the cantilever. Another way can be to simply pull on the free end of the cantilever with a mechanical drive. Whichever method is used to bend the cantilever, it is important that the proper curvature be used in the geometric model. Because an end-moment type deflection (cantilever sensors) does not bend the cantilever in the same way as a point load (AFM force sensing) (see Fig. 3 in Ref. 9), both will give a different optimized setup. However, once all the parameters $(D, L, \mathrm{CL}, \phi, \theta$, and $\xi)$ are determined, the exact relationship between the cantilever deflection and PSD signal can be determined with absolute accuracy for any kind of cantilever bending even if the PSD signal/cantilever deflection relationship is no longer linear.

Having an accurate PSD signal/cantilever deflection calibration is especially important when performing differential cantilever sensor measurements. ${ }^{16,17}$ In such experiments, it is necessary to subtract the signals of two (or more) cantilever deflections in order to isolate the measurement of a specific physical or chemical phenomenon. Error in the cantilever deflections will lead to an increased error on the measured physical quantity.

In this letter we have shown how our model can be used to improve the design of the next generation of cantileverbased sensors and atomic force microscopes. Using this model to optimize the system's geometry promises to significantly simplify the use of such instruments. Moreover, due to the accuracy of this model, it can also be used to obtain an exact numerical relationship between the PSD signal and cantilever deflection of any existing system for any kind of cantilever deflection provided a method is found to measure the parameters $D, L, \mathrm{CL}, \phi, \theta$, and $\xi$ with sufficient accuracy.

The authors would like to thank the Natural Sciences and Engineering Research Council of Canada (NSERC), Genome Quebec, and McGill University for funding through various grants and fellowships.

${ }^{1}$ G. Meyer and N. M. Amer, Appl. Phys. Lett. 53, 2400 (1988).

${ }^{2}$ T. A. Brunt, E. D. Chabala, T. Rayment, S. J. O'Shea, and M. E. Welland, J. Chem. Soc., Faraday Trans. 92, 3807 (1996).

${ }^{3}$ A. García-Valenzuela, J. Appl. Phys. 82, 985 (1997).

${ }^{4}$ A. García-Valenzuela and J. Villatoro, J. Appl. Phys. 84, 58 (1998).

${ }^{5}$ P. G. Datskos, S. Rajic, and I. Datskou, Appl. Phys. Lett. 73, 2319 (1998).

${ }^{6}$ S. J. O'Shea, M. E. Welland, T. A. Brunt, A. R. Ramadan, and T. Rayment, J. Vac. Sci. Technol. B 14, 1383 (1996).

${ }^{7}$ G. Moulard, G. Contoux, G. Gardet, G. Motyl, and M. Courbon, J. Vac. Sci. Technol. A 16, 736 (1997).

${ }^{8}$ N. P. D’Costa and J. H. Hoh, Rev. Sci. Instrum. 66, 5096 (1995).

${ }^{9}$ T. Miyatani and M. Fujihira, J. Appl. Phys. 81, 7099 (1997).

${ }^{10}$ Z. Hu, T. Seeley, S. Kossek, and T. Thundat, Rev. Sci. Instrum. 75, 400 (2004).

${ }^{11}$ M. Godin, V. Tabard-Cossa, P. Grütter, and P. J. Williams, Appl. Phys. Lett. 79, 551 (2001).

${ }^{12}$ G. E. Farin and D. Hansford, The Geometry Toolbox for Graphics and Modeling (Natick, MA, 1998).

${ }^{13}$ G. G. Stoney, Proc. R. Soc. London, Ser. A 82, 172 (1909).

${ }^{14}$ R. W. Hoffman, Physics of Thin Films 3 (Academic, New York, 1966).

${ }^{15}$ L. Y. Beaulieu, M. Godin, O. Laroche, V. Tabard-Cossa, and P. Grütter (unpublished).

${ }^{16}$ M. Godin, O. Laroche, V. Tabard-Cossa, L. Y. Beaulieu, P. Grütter, and P. J. Williams, Rev. Sci. Instrum. 74, 4902 (2003).

${ }^{17}$ H. P. Lang, R. Berger, F. Battiston, J.-P. Ramseyer, E. Meyer, C. Andreoli, J. Brugger, P. Vettiger, M. Despont, T. Mezzacasa, L. Scandella, H.-J. Gntherodt, C. Gerber, and J. K. Gimzewski, Appl. Phys. A: Mater. Sci. Process. 66, S61 (1998). 